\title{
Almost-Periodic Weak Solutions of Second-Order Neutral Delay-Differential Equations with Piecewise Constant Argument
}

\author{
Li Wang and Chuanyi Zhang \\ Department of Mathematics, Harbin Institute of Technology, Harbin 150001, China \\ Correspondence should be addressed to Li Wang, wanglimath@yahoo.com.cn \\ Received 10 December 2007; Accepted 4 June 2008 \\ Recommended by Ondrej Dosly
}

We investigate the existence of almost-periodic weak solutions of second-order neutral delaydifferential equations with piecewise constant argument of the form $(x(t)+x(t-1))^{\prime \prime}=q x(2[(t+$ $1) / 2])+f(t)$, where [·] denotes the greatest integer function, $q$ is a real nonzero constant, and $f(t)$ is almost periodic.

Copyright (C) 2008 L. Wang and C. Zhang. This is an open access article distributed under the Creative Commons Attribution License, which permits unrestricted use, distribution, and reproduction in any medium, provided the original work is properly cited.

\section{Introduction and preliminaries}

Differential equations with piecewise constant argument, which were firstly considered by Cooke and Wiener [1], and Shah and Wiener [2], usually describe hybrid dynamical systems (a combination of continuous and discrete) and so combine properties of both differential and difference equations. Over the years, great attention has been paid to the study of the existence of almost-periodic-type solutions of this type of equations. There are many remarkable works on this field (see [3-10] and references therein). Particularly, the second-order neutral delaydifferential equations with piecewise constant argument of the form

$$
(x(t)+p x(t-1))^{\prime \prime}=q x\left(2\left[\frac{t+1}{2}\right]\right)+f(t)
$$

have been intensively studied for $|p| \neq 1$ by different methods, where [·] denotes the greatest integer function, $p, q$ are real nonzero constants, and $f(t)$ is almost periodic. In [6], Li introduced the concepts of odd-weak solution, even-weak solution, and weak solution of (1.1). Some theorems about the existence of almost-periodic weak solutions were obtained while 
putting restriction on the function $f$. Papers $[7,8]$ concentrated on dealing with the existence and uniqueness of pseudo-almost-periodic solution by putting some restrictions on the roots of characteristic equation instead of on the function $f$. If $f(t)$ is replaced by a nonlinear function $g(t, x(t), x([t]))$, some results about the existence and uniqueness of almost-periodic solution or pseudo-almost-periodic solution were obtained in [8-10].

Up to now, there have been no papers concerning the solutions or weak solutions of (1.1) when $p=1$. In this paper, we study this case, namely, the equation

$$
(x(t)+x(t-1))^{\prime \prime}=q x\left(2\left[\frac{t+1}{2}\right]\right)+f(t) .
$$

In constructing almost-periodic-type solution or weak solution of (1.1) in [6-10], the condition $|p| \neq 1$ is essential because it guarantees the convergence of the related series. To investigate such equation as (1.2), we have to give a quite different consideration.

Now we give some definitions. Throughout this paper, $\mathcal{Z}, \mathcal{R}$, and $\mathcal{C}$ denote the sets of integers, real, and complex numbers, respectively. The following definitions can be found in any book, say [11], on almost-periodic functions.

Definition 1.1. (1). A subset $P$ of $\mathcal{R}$ is said to be relatively dense in $\mathcal{R}$ if there exists a number $p>0$ such that $P \cap[t, t+p] \neq \varnothing$ for all $t \in \mathcal{R}$.

(2). A continuous function $f: \mathcal{R} \rightarrow \mathcal{R}$ is called almost periodic (abbreviated as $A D(R)$ ) if the $\epsilon$-translation set of $f$

$$
T(f, \epsilon)=\{\tau \in \mathcal{R}:|f(t+\tau)-f(t)|<\epsilon, \forall t \in \mathcal{R}\}
$$

is relatively dense for each $\epsilon>0$.

Definition 1.2. (1) For a sequence $\{x(n): n \in Z\}$, define $[x(n), x(n+p)]=\{x(n), \ldots, x(n+p)\}$ and call it sequence interval with length $p$. A subset $P$ of $z$ is said to be relatively dense in $z$ if there exists a positive integer $p$ such that $P \cap[n, n+p] \neq \varnothing$ for all $n \in \mathcal{Z}$.

(2) A bounded sequence $x: Z \rightarrow R$ (resp., $\mathcal{C}$ ) is called an almost-periodic sequence (abbreviated as $A D S(R)$ ) (resp., abbreviated as $A D S(C)$ ) if the $\epsilon$-translation set of $x$

$$
T(x, \epsilon)=\{\tau \in \mathfrak{Z}:|x(n+\tau)-x(n)|<\epsilon, \forall n \in \mathfrak{Z}\}
$$

is relatively dense for each $\epsilon>0$.

As mentioned in [6], we have the following definitions.

Definition 1.3. A continuous function $x: R \rightarrow R$ is called an odd-weak solution (resp., evenweak solution) of (1.2) if the following conditions are satisfied:

(i) $x(t)$ satisfies (1.2) for $t \in R, t \neq n \in z$;

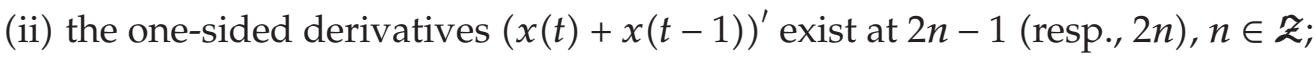

(iii) the one-sided second-order derivatives $(x(t)+x(t-1))^{\prime \prime}$ exist at $2 n$ (resp., $\left.2 n-1\right)$, $n \in \mathfrak{z}$. 
Both odd-weak solution (abbreviated as ow-solution) and even-weak solution (abbreviated as ew-solution) of (1.2) are called weak solution (abbreviated as w-solution) of (1.2). It should be pointed out that if $x(t)$ is an ow-solution (resp., ew-solution) of (1.2), then $(x(t)+x(t-1))^{\prime}$ are continuous at $2 n$ (resp., $\left.2 n-1\right), n \in z$; ow-solution of (1.2) is not equivalent to ew-solution of $(1.2) ; x(t)$ is a solution of (1.2) if it is an ow-solution as well as an ew-solution of (1.2).

Let $p_{1}(\lambda)=\lambda^{3}-(1+q) \lambda^{2}-\lambda+1$ and $p_{2}(\lambda)=(1-q / 2) \lambda^{3}-\lambda^{2}-(1+q / 2) \lambda+1$, then the following hold.

Lemma 1.4. Assume $q \neq 0$. The roots of polynomials $p_{i}(\lambda)$ are of modules different from $1, i=1,2$.

Proof. It is clear that 1 and -1 are not the roots of $p_{i}(\lambda)$ because $q \neq 0, i=1,2$. Denote the three roots of $p_{1}(\lambda)$ by $\lambda_{1}, \lambda_{2}, \lambda_{3} \in \mathcal{C}$, without loss of generality, let $\lambda_{1}=e^{i \theta}, \lambda_{2}=e^{-i \theta}$, here $\theta$ is a real constant, thus we obtain $\lambda_{3}=-1$, which is impossible. So, the modules of roots of polynomial $p_{1}(\lambda)$ are not 1 .

If $q=2$, it is obvious that the result holds for $p_{2}(\lambda)$. If $q \neq 2$, denote the three roots of $p_{2}(\lambda)$ by $\lambda_{1}^{\prime}, \lambda_{2}^{\prime}, \lambda_{3}^{\prime} \in \mathcal{C}$, without loss of generality, let $\lambda_{1}^{\prime}=e^{i \theta}, \lambda_{2}^{\prime}=e^{-i \theta}$, here $\theta$ is a real constant, then we have

$$
\begin{gathered}
\lambda_{1}^{\prime} \lambda_{2}^{\prime}=1, \quad \lambda_{1}^{\prime} \lambda_{2}^{\prime} \lambda_{3}^{\prime}=\frac{-1}{(1-q / 2)}, \\
\lambda_{1}^{\prime}+\lambda_{2}^{\prime}+\lambda_{3}^{\prime}=\frac{1}{(1-q / 2)}, \\
\lambda_{1}^{\prime} \lambda_{2}^{\prime}+\lambda_{1}^{\prime} \lambda_{3}^{\prime}+\lambda_{2}^{\prime} \lambda_{3}^{\prime}=-\frac{(1+q / 2)}{(1-q / 2)} .
\end{gathered}
$$

This implies that $q=0$ and contradicts the hypothesis. The proof is complete.

The rest of this paper is organized as follows. Section 2 is devoted to the main theorems and their proofs. In Section 3, some examples are given to explain our results and illuminate the relationship among solution, ow-solution, and ew-solution.

\section{The main results}

Let

$$
f_{n}^{(1)}=\int_{n}^{n+1} \int_{n}^{s} f(\sigma) d \sigma d s, \quad f_{n}^{(2)}=\int_{n}^{n-1} \int_{n}^{s} f(\sigma) d \sigma d s, \quad h_{n}=f_{n}^{(1)}+f_{n}^{(2)} .
$$

To present the main results of this paper, we need the following assumption.

(H) $f(t) \in \mathcal{A} D(R)$ is such that there exists $g(t) \in \mathcal{A} D(R)$ such that $f(t)=g(t)-g(t-2)$, for all $t \in \mathcal{R}$.

Remark 2.1. (1) $\mathscr{A} D(R)$ is a translation invariant Banach space. For every $g \in \mathcal{A D}(R)$, one has $g(\cdot-2) \in \mathcal{A} D(R)$ too. Set $f(t)=g(t)-g(t-2)$, then $f$ satisfies $(\mathrm{H})$, and therefore there exist a great number of functions satisfying the assumption $(\mathrm{H}) .(2)$ Reference [5] uses an assumption similar to $(\mathrm{H})$ implicitly.

Let $E=\left\{\left\{a_{n}\right\} \in \mathcal{A} P \mathcal{S}(R): a_{n}=\widehat{a}_{n+2}-\widehat{a}_{n}\right.$, for all $\left.n \in \mathcal{Z},\left\{\widehat{a}_{n}\right\} \in \mathcal{A} D \mathcal{S}(R)\right\}$. We have the following lemma. 
Lemma 2.2. Under the assumption $(H)$, one has $\left\{f_{n}^{(1)}\right\},\left\{f_{n}^{(2)}\right\},\left\{h_{n}\right\} \in E$.

Proof. By $(\mathrm{H})$, there exists $g(t) \in \mathcal{A} P(R)$ such that $f(t)=g(t)-g(t-2), t \in \mathcal{R}$. Let $\widehat{f}_{n+2}^{(1)}=$ $g_{n}^{(1)}=\int_{n}^{n+1} \int_{n}^{s} g(\sigma) d \sigma d s$ and $\widehat{f}_{n+2}^{(2)}=g_{n}^{(2)}=\int_{n}^{n-1} \int_{n}^{s} g(\sigma) d \sigma d s$, it is easy to verify that $\left\{f_{n}^{(1)}\right\},\left\{f_{n}^{(2)}\right\}$, $\left\{h_{n}\right\},\left\{g_{n}^{(1)}\right\},\left\{g_{n}^{(2)}\right\},\left\{\widehat{f}_{n}^{(1)}\right\},\left\{\widehat{f}_{n}^{(2)}\right\} \in \mathcal{A D S}(R)$, and $f_{n}^{(1)}=g_{n}^{(1)}-g_{n-2}^{(1)}=\widehat{f}_{n+2}^{(1)}-\widehat{f}_{n}^{(1)}, f_{n}^{(2)}=g_{n}^{(2)}-g_{n-2}^{(2)}=$ $\widehat{f}_{n+2}^{(2)}-\widehat{f}_{n}^{(2)}$, for all $n \in \mathcal{Z}$, that is, $\left\{f_{n}^{(1)}\right\},\left\{f_{n}^{(2)}\right\} \in E$. Set $\widehat{h}_{n+2}=g_{n}=g_{n}^{(1)}+g_{n}^{(2)}$, similarly we can obtain $\left\{\widehat{h}_{n}\right\},\left\{g_{n}\right\} \in \mathcal{A} \mathcal{S S}(R)$, and $h_{n}=\widehat{h}_{n+2}-\widehat{h}_{n}=g_{n}-g_{n-2}$, for all $n \in \mathcal{Z}$, that is, $\left\{h_{n}\right\} \in E$.

Lemma 2.3. Suppose that $X$ is a Banach space, $\mathcal{L}(X)$ denotes the set of bounded linear operators from $X$ to $X, A \in \mathcal{L}(X)$ and $\|A\|<1$, then $I d-A$ is bounded invertible and

$$
\begin{gathered}
(I d-A)^{-1}=\sum_{n=0}^{\infty} A^{n}, \\
\left\|(I-A)^{-1}\right\| \leq \frac{1}{(1-\|A\|)},
\end{gathered}
$$

where $A^{0}=I d, I d$ is an identical operator.

The proof of Lemma 2.3 can be found in any book of functional analysis. We remark that if $A$ is a linear operator and its inverse exists, then $A^{-1}$ is also a linear operator.

To get w-solutions or solutions of (1.2), we start with its corresponding difference equations.

Suppose that $x(t)$ is an ow-solution of (1.2), then $x(t)$ satisfies the three conditions in Definition 1.3. By a process of integrating (1.2) two times in $t \in[2 n-1,2 n+1)$ as in [6-10], we can easily get

$$
x(2 n+1)-(1+q) x(2 n)-x(2 n-1)+x(2 n-2)=h_{2 n}
$$

Similarly if $x(t)$ is an ew-solution of (1.2), by the process of integrating (1.2) two times in $t \in[2 n-2,2 n)$, we get

$$
\left(1-\frac{q}{2}\right) x(2 n)-x(2 n-1)-\left(1+\frac{q}{2}\right) x(2 n-2)+x(2 n-3)=h_{2 n-1}
$$

These lead to the difference equations

$$
\begin{gathered}
x_{2 n+1}-(1+q) x_{2 n}-x_{2 n-1}+x_{2 n-2}=h_{2 n} \\
\left(1-\frac{q}{2}\right) x_{2 n}-x_{2 n-1}-\left(1+\frac{q}{2}\right) x_{2 n-2}+x_{2 n-3}=h_{2 n-1} .
\end{gathered}
$$

From the analysis above, one sees that if $x(t)$ is an ow-solution (resp., ew-solution) of (1.2), then one gets (2.5) (resp., (2.6)); if $x(t)$ is a solution of (1.2), then one gets both (2.5) and (2.6). Conversely, we will show that the w-solutions or solutions of (1.2) are obtained via the solutions of (2.5) and (2.6). In order to get the solutions of (2.5) and (2.6), we will consider the 
following difference equations:

$$
\begin{gathered}
x_{n+1}-(1+q) x_{n}-x_{n-1}+x_{n-2}=h_{n} \\
\left(1-\frac{q}{2}\right) x_{n}-x_{n-1}-\left(1+\frac{q}{2}\right) x_{n-2}+x_{n-3}=h_{n-1}
\end{gathered}
$$

Notice that for any sequences $\left\{a_{n}\right\},\left\{b_{n}\right\}$, and $\alpha, \beta \in \mathcal{R}$, one has $\alpha\left\{a_{n}\right\}+\beta\left\{b_{n}\right\}=\left\{c_{n}: c_{n}=\right.$ $\left.\alpha a_{n}+\beta b_{n}, n \in \mathfrak{z}\right\}$. Especially, $\left\{a_{n}\right\}=\left\{b_{n}\right\} \Leftrightarrow a_{n}=b_{n}$, for all $n \in \mathfrak{z}$. In virtue of studying (2.7) and (2.8), we have the following theorem.

Theorem 2.4. Under the assumption (H), (2.7) (resp., (2.8)) has a unique solution $\left\{x_{n}\right\} \in E$ (resp., $\left.\left\{y_{n}\right\} \in E\right)$.

Proof. As the proof of [7, Theorem 9], define $A: X \rightarrow X$ by $A\left\{x_{n}\right\}=\left\{x_{n+1}\right\}$, where $X$ is the Banach space consisting of all bounded sequences $\left\{x_{n}\right\}$ in $\mathcal{C}$ with $\left\|\left\{x_{n}\right\}\right\|=\sup _{n \in \mathcal{Z}}\left|x_{n}\right|$. Notice Lemmas 1.4 and 2.3, we know that (2.7) has a unique solution $\left\{x_{n}\right\}=p_{1}(A)^{-1}\left\{h_{n+2}\right\} \in \mathcal{A} P \mathcal{S}(R)$. By the process of proving Lemma 2.2, we have $\left\{x_{n}\right\}=p_{1}(A)^{-1}\left\{h_{n+2}\right\}=p_{1}(A)^{-1}\left\{g_{n+2}\right\}-$ $p_{1}(A)^{-1}\left\{g_{n}\right\}=\left\{\widehat{x}_{n+2}\right\}-\left\{\widehat{x}_{n}\right\}$, that is, $x_{n}=\widehat{x}_{n+2}-\widehat{x}_{n}$, for all $n \in \mathcal{Z}$, where $\left\{\widehat{x}_{n}\right\}=p_{1}(A)^{-1}\left\{g_{n}\right\} \in$ $\operatorname{ADS}(R)$ (this follows in the same way as [7, Theorem 9]). Therefore, (2.7) has a unique solution $\left\{x_{n}\right\} \in E$.

Similarly, (2.8) has a unique solution $\left\{y_{n}\right\}=p_{2}(A)^{-1}\left\{h_{n+2}\right\} \in \operatorname{ADS}(R)$ and $\left\{y_{n}\right\}=$ $p_{2}(A)^{-1}\left\{h_{n+2}\right\}=p_{2}(A)^{-1}\left\{g_{n+2}\right\}-p_{2}(A)^{-1}\left\{g_{n}\right\}=\left\{\widehat{y}_{n+2}\right\}-\left\{\widehat{y}_{n}\right\}$, that is, $y_{n}=\widehat{y}_{n+2}-\widehat{y}_{n}$, for all $n \in$ $\mathcal{z}$, where $\left\{\widehat{y}_{n}\right\}=p_{2}(A)^{-1}\left\{g_{n}\right\} \in \mathcal{A} \mathcal{S}(R)$. Therefore, (2.8) has a unique solution $\left\{y_{n}\right\} \in E$. This completes the proof.

Remark 2.5. (i) In Theorem 2.4, since $\left\{\widehat{x}_{n}\right\}=p_{1}(A)^{-1}\left\{g_{n}\right\}$ and $\left\{\widehat{y}_{n}\right\}=p_{2}(A)^{-1}\left\{g_{n}\right\}$, we can easily get

$$
\begin{gathered}
\widehat{x}_{2 n+3}-(1+q) \widehat{x}_{2 n+2}-\widehat{x}_{2 n+1}+\widehat{x}_{2 n}=g_{2 n} \\
\left(1-\frac{q}{2}\right) \widehat{y}_{2 n}-\widehat{y}_{2 n-1}-\left(1+\frac{q}{2}\right) \widehat{y}_{2 n-2}+\widehat{y}_{2 n-3}=g_{2 n-3} .
\end{gathered}
$$

It must be stressed that (2.9) and (2.10) are important, since they can guarantee the continuity of the w-solutions or solutions of (1.2) constructed in Theorems 2.6, 2.7, and 2.8.

(ii) Let $F_{1}=\left\{\left\{a_{n}\right\}:\left\{a_{n}\right\} \in E\right.$ with $\left\{\widehat{a}_{n}\right\} \in \mathcal{A D S}(R)$ satisfying (2.9) $\}$, and $F_{2}=\left\{\left\{b_{n}\right\}\right.$ : $\left\{b_{n}\right\} \in E$ with $\left\{\widehat{b}_{n}\right\} \in \operatorname{APS}(R)$ satisfying (2.10) $\}$. Notice the fact that the solution of (2.7) (resp., (2.8)) must be a solution of (2.5) (resp., (2.6)), it is false conversely. So, suppose the assumption (H) holds, it follows from Theorem 2.4 that (2.5) (resp., (2.6)) has solution $\left\{x_{n}\right\} \in$ $F_{1}$ (resp., $\left\{y_{n}\right\} \in F_{2}$ ). Moreover, such solutions may not be unique. See Example 3.1 at the end of this paper.

In the following, we focus on seeking the almost-periodic w-solutions or solutions of (1.2) via the almost-periodic sequence solutions of (2.5) and (2.6). As mentioned above, it is due to $p=1$ that, to get almost-periodic w-solutions or solutions of (1.2), we have to use a way quite different from [6-10]. Our main idea is to construct solutions or w-solutions of (1.2) piecewise. It seems that this is a new technique. 
Without loss of generality, suppose that $\left\{x_{n}\right\} \in F_{1}$ (resp., $\left\{y_{n}\right\} \in F_{2}$ ) is an arbitrary solution of (2.5) (resp., (2.6)). To prove the following theorems, we need to introduce some notations firstly:

$$
\begin{aligned}
\omega_{2 n} & =x_{2 n+1}-\frac{q}{2} x_{2 n}-x_{2 n-1}-f_{2 n}^{(1)} \\
& =\widehat{x}_{2 n+3}-\frac{q}{2} \widehat{x}_{2 n+2}-\widehat{x}_{2 n+1}-g_{2 n}^{(1)}-\left(\widehat{x}_{2 n+1}-\frac{q}{2} \widehat{x}_{2 n}-\widehat{x}_{2 n-1}-g_{2 n-2}^{(1)}\right) \\
& =\widehat{\omega}_{2 n+2}-\widehat{\omega}_{2 n} ; \\
\omega_{2 n-1} & =\left(1-\frac{q}{2}\right) y_{2 n}-y_{2 n-2}-f_{2 n-1}^{(1)} \\
& =\left(1-\frac{q}{2}\right) \widehat{y}_{2 n+2}-\widehat{y}_{2 n}-g_{2 n-1}^{(1)}-\left(\left(1-\frac{q}{2}\right) \widehat{y}_{2 n}-\widehat{y}_{2 n-2}-g_{2 n-3}^{(1)}\right) \\
& =\widehat{\omega}_{2 n+1}-\widehat{\omega}_{2 n-1},
\end{aligned}
$$

where $\widehat{\omega}_{2 n}=\widehat{x}_{2 n+1}-q \widehat{x}_{2 n} / 2-\widehat{x}_{2 n-1}-g_{2 n-2}^{(1)}$ and $\widehat{\omega}_{2 n-1}=(1-q / 2) \widehat{y}_{2 n}-\widehat{y}_{2 n-2}-g_{2 n-3}^{(1)}$. It can be easily verified that $\left\{\omega_{2 n}\right\},\left\{\omega_{2 n-1}\right\},\left\{\widehat{\omega}_{2 n}\right\},\left\{\widehat{\omega}_{2 n-1}\right\} \in \mathcal{A D S}(R)$.

For the existence of the almost-periodic ow-solution of (1.2), we have the following.

Theorem 2.6. Under the assumption $(H),(1.2)$ has an ow-solution $x(t) \in \mathcal{A} D(R)$ such that $x(n)=$ $x_{n}$, for all $n \in \mathcal{z}$.

Proof. Under the assumption $(\mathrm{H})$, define $x(t)$ as

$$
x(t)= \begin{cases}x_{1}(t), & t \in[2 n, 2 n+1) \forall n \in Z, \\ x_{2}(t), & t \in[2 n-1,2 n) \forall n \in Z,\end{cases}
$$

where

$$
\begin{aligned}
x_{1}(t)= & \widehat{\omega}_{2 n+2}+q \widehat{x}_{2 n+2}\left(t-2 n-\frac{1}{2}\right)+\int_{t-1}^{t} \int_{2 n}^{s} g(\sigma) d \sigma d s, \quad t \in[2 n, 2 n+1), \forall n \in Z, \\
x_{2}(t)= & x_{2 n}+x_{2 n-1}+\widehat{\omega}_{2 n+2}(t-2 n)-\widehat{\omega}_{2 n}(t+1-2 n)+\frac{q}{2} \widehat{x}_{2 n+2}(t-2 n)^{2}-\frac{q}{2} \widehat{x}_{2 n}(t+1-2 n)^{2} \\
& +\int_{2 n}^{t} \int_{2 n}^{s} g(\sigma) d \sigma d s-\int_{2 n-2}^{t-1} \int_{2 n-2}^{s} g(\sigma) d \sigma d s, \quad t \in[2 n-1,2 n), \quad \forall n \in Z .
\end{aligned}
$$

From (2.9), it follows that $x(t)$ is continuous on $\mathcal{R}$ and $x(n)=x_{1}(n)=x_{2}(n)=x_{n}, n \in \mathcal{z}$. Moreover, for $t \in[2 n, 2 n+1), n \in \mathfrak{Z}$, one has $x(t)+x(t-1)=x_{1}(t)+x_{2}(t-1)$; for $t \in[2 n-1,2 n)$, $n \in \mathfrak{z}$, one has $x(t)+x(t-1)=x_{2}(t)+x_{1}(t-1)$. By simple calculation, for $t \in[2 n-1,2 n+1)$, $n \in Z$, we have

$$
x(t)+x(t-1)=x_{2 n}+x_{2 n-1}+\omega_{2 n}(t-2 n)+\frac{q}{2} x_{2 n}(t-2 n)^{2}+\int_{2 n}^{t} \int_{2 n}^{s} f(\sigma) d \sigma d s .
$$

Note that $(x(t)+x(t-1))^{\prime}=\omega_{2 n}+q x_{2 n}(t-2 n)+\int_{2 n}^{t} f(s) d s$, this implies that the one-sided derivatives $(x(t)+x(t-1))^{\prime}$ exist at $2 n-1, n \in \mathcal{~ z . ~ S i n c e ~}(x(t)+x(t-1))^{\prime \prime}=q x_{2 n}+f(t)=$ $q x(2[(t+1) / 2])+f(t)$, the second-order derivatives $(x(t)+x(t-1))^{\prime \prime}$ are continuous at $2 n$,

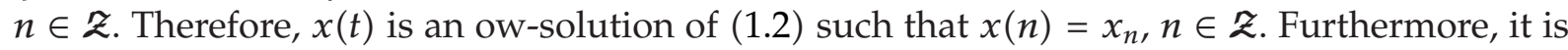
easy to check that $x(t)$ is almost periodic, we omit the details. The proof is complete. 
L. Wang and C. Zhang

For the existence of the almost-periodic ew-solution of (1.2), we have the following.

Theorem 2.7. Under the assumption $(H),(1.2)$ has an ew-solution $y(t) \in \mathcal{A} P(R)$ such that $y(n)=$ $y_{n}$, for all $n \in \mathcal{z}$.

Proof. Under the assumption $(\mathrm{H})$, define $y(t)$ as

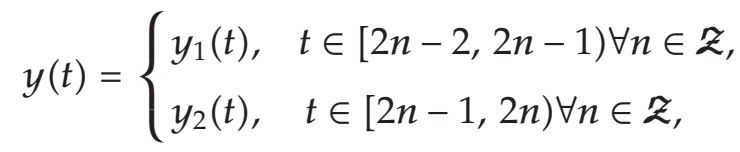

where

$$
\begin{aligned}
y_{1}(t)= & y_{2 n-1}+y_{2 n-2}+\widehat{\omega}_{2 n+1}(t-2 n+1)-\widehat{\omega}_{2 n-1}(t+2-2 n)+\frac{q}{2} \widehat{y}_{2 n}(t-2 n+1)^{2} \\
& -\frac{q}{2} \widehat{y}_{2 n}(t+2-2 n)^{2}+\int_{2 n-1}^{t} \int_{2 n-1}^{s} g(\sigma) d \sigma d s-\int_{2 n-3}^{t-1} \int_{2 n-3}^{s} g(\sigma) d \sigma d s, \quad t \in[2 n-2,2 n-1), \\
y_{2}(t)= & \widehat{\omega}_{2 n+1}+\frac{q}{2} \widehat{y}_{2 n+2}(t-2 n+1)^{2}-\frac{q}{2} \widehat{y}_{2 n}(t-2 n)^{2}+\int_{t-1}^{t} \int_{2 n-1}^{s} g(\sigma) d \sigma d s, \quad t \in[2 n-1,2 n) .
\end{aligned}
$$

From (2.10), it follows that $y(t)$ is continuous on $\mathcal{R}$ and $y(n)=y_{1}(n)=y_{2}(n)=y_{n}, n \in \mathfrak{z}$. The rest of the proof is similar to that of Theorem 2.6, we omit the details.

For the existence of almost-periodic solution of (1.2), we have the following.

Theorem 2.8. Under the assumption $(H)$, if $\left\{x_{n}\right\} \in F_{1} \cap F_{2}$ is the common solution of (2.5) and (2.6), then (1.2) has a solution $x(t) \in \mathcal{A D}(R)$ such that $x(n)=x_{n}, n \in \mathcal{Z}$. If $\left\{y_{n}\right\}$ replaces $\left\{x_{n}\right\}$, the conclusion is still true.

Proof. Since $\left\{x_{n}\right\} \in F_{1} \cap F_{2}$ and $\left\{\widehat{x}_{n}\right\} \in \mathcal{A} P \mathcal{S}(R)$ are solutions of (2.5) and (2.9) respectively, and they are also solutions of (2.6) and (2.10), respectively, it follows from Theorems 2.6 and 2.7 that, by simple calculation, the almost-periodic ow-solution $x(t)$ constructed as the proof of Theorem 2.6 with $x(n)=x_{n}, n \in \mathcal{Z}$, is the same as the almost-periodic ew-solution $y(t)$ constructed as the proof of Theorem 2.7 with $y(n)=x_{n}, n \in z$. This implies $x(t)$ is an almostperiodic solution of (1.2) such that $x(n)=x_{n}, n \in \mathcal{Z}$. If $\left\{y_{n}\right\}$ replaces $\left\{x_{n}\right\}$, the proof is similar, we omit the details.

Remark 2.9. As mentioned above, an ow-solution of (1.2) is not equivalent to an ew-solution of (1.2), and a solution of (1.2) is an ow-solution of (1.2) as well as an ew-solution of (1.2). See the examples in Section 3.

The following theorem is usually used for judging whether or not a w-solution of (1.2) is a solution of (1.2).

Theorem 2.10. Suppose that $x(t)$ is a solution of (1.2), then

$$
\left(1-\frac{q}{2}\right) x(2 n+2)-\left(2+\frac{3 q}{2}\right) x(2 n)+x(2 n-2)=h_{2 n}+h_{2 n+1} .
$$


Proof. If $x(t)$ is a solution of (1.2), then $\{x(n)\}$ must be common solution of (2.5) and (2.6). Moreover, (2.6) is equivalent to

$$
\left(1-\frac{q}{2}\right) x_{2 n+2}-x_{2 n+1}-\left(1+\frac{q}{2}\right) x_{2 n}+x_{2 n-1}=h_{2 n+1}
$$

Substituting $x(n)$ into both the above equation and (2.5), then add the resulting equations to get the result.

\section{Some examples}

In this section, we first explain how to get almost periodic w-solutions and solutions of (1.2) specifically. And then, we present two examples: in Example 3.1, we aim mainly to obtain the almost-periodic solution, and in Example 3.2, we obtain the almost-periodic ow-solution and ew-solution. Consequently, the relationship among ow-solution, ew-solution, and solution is shown. Besides, Example 3.1 also illuminates that the solutions in $F_{1}$ (resp., $F_{2}$ ) of (2.5) (resp., (2.6)) may not be unique.

Under the assumption $(\mathrm{H})$, it follows from the proof of Theorem 2.6 (resp., 2.7) that we can get almost-periodic ow-solution (resp., ew-solution) of (1.2) by the following three steps.

(i) Calculate $f_{n}^{(1)}, f_{n}^{(2)}, h_{n}, g_{n}^{(1)}, g_{n}^{(2)}, g_{n}, \int_{t-1}^{t} \int_{n}^{s} g(\sigma) d \sigma d s, t \in[n, n+1), n \in \mathcal{Z}$, and $\int_{n}^{t} \int_{n}^{s} g(\sigma) d \sigma d s-\int_{n-2}^{t-1} \int_{n-2}^{s} g(\sigma) d \sigma d s, t \in[n-1, n), n \in \mathfrak{z}$.

(ii) Seek the solution $\left\{x_{n}\right\} \in F_{1}$ (resp., $\left\{y_{n}\right\} \in F_{2}$ ) of (2.5) (resp., (2.6)). Calculate $\omega_{2 n}$ and $\widehat{\omega}_{2 n}$ (resp., $\omega_{2 n+1}$ and $\widehat{\omega}_{2 n+1}$ ).

(iii) By the proof of Theorem 2.6 (resp., 2.7), we get the almost-periodic ow-solution $x(t)$ (resp., ew-solution $y(t))$ such that $x(n)=x_{n},\left(\right.$ resp., $\left.y(n)=y_{n}\right), n \in \mathfrak{z}$.

On the other hand, it follows from the proof of Theorem 2.8 that we can get the almostperiodic solution by the following steps.

(i) Seek the solution $\left\{x_{n}\right\}$ in $F_{1} \cap F_{2}$ which is the common solution of (2.5) and (2.6). Calculate $\omega_{2 n}, \widehat{\omega}_{2 n}, \omega_{2 n+1}$, and $\widehat{\omega}_{2 n+1}$.

(ii) Find the almost periodic ow-solution $x(t)$ such that $x(n)=x_{n}, n \in z$ or ew-solution $y(t)$ such that $y(n)=x_{n}, n \in z$ by the above methods. From Theorem 2.8, we know they are the same, that is, it must be the almost periodic solution.

The following example shows that a solution of (1.2) is an ow-solution of (1.2) as well as an ew-solution of (1.2), and the solutions in $F_{1}$ (resp., $F_{2}$ ) of (2.5) (resp., (2.6)) may not unique.

Example 3.1. Let $q=2, f(t)=\cos (\pi t / 2)$, and $g(t)=\cos (\pi t / 2) / 2$, then $f(t), g(t) \in \mathcal{A} D(R)$, and $f(t)=g(t)-g(t-2)$, for all $t \in R$, that is, $f(t)$ satisfy the assumption (H). By simple calculation, we can obtain $f_{n}^{(1)}=(2 / \pi)^{2}(\sin (\pi n / 2)+\cos (\pi n / 2))-2 \sin (\pi n / 2) / \pi, g_{n}^{(1)}=f_{n}^{(1)} / 2, f_{n}^{(2)}=$ $(2 / \pi)^{2}(\cos (\pi n / 2)-\sin (\pi n / 2))+2 \sin (\pi n / 2) / \pi, g_{n}^{(2)}=f_{n}^{(2)} / 2$, and $g_{2 n}=h_{2 n} / 2=(-1)^{n} 4 / \pi^{2}$, $g_{2 n+1}=h_{2 n+1} / 2=0$, for all $n \in \mathcal{z}$. 
(i) We construct the almost-periodic solution of (1.2) as the proof of Theorem 2.8. Let

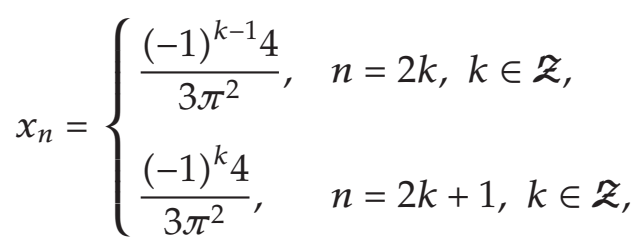

$$
\begin{aligned}
& \widehat{x}_{n}= \begin{cases}\frac{(-1)^{k} 2}{3 \pi^{2}}, & n=2 k, k \in \mathcal{Z}, \\
\frac{(-1)^{k-1} 2}{3 \pi^{2}}, & n=2 k+1, k \in \mathfrak{Z},\end{cases}
\end{aligned}
$$

then $\left\{x_{n}\right\} \in F_{1} \cap F_{2}$ is the common solution of (2.5) and (2.6). Calculate $\widehat{\omega}_{2 n}, \widehat{\omega}_{2 n+1}, \omega_{2 n}, \omega_{2 n-1}$ by the formulas mentioned above, we obtain $\widehat{\omega}_{2 n}=\omega_{2 n}=0, \widehat{\omega}_{2 n+1}=(-1)^{n+1}(3 \pi-4) / 3 \pi^{2}$, $\omega_{2 n-1}=(-1)^{n+1}(6 \pi-8) / 3 \pi^{2}$. Obviously, $\left\{\omega_{n}\right\} \in E$ and $\left\{\widehat{\omega}_{n}\right\} \in \mathcal{A} \mathcal{S}(R)$. Define the ow-solution and the ew-solution as the proofs of Theorems 2.6 and 2.7, respectively, it follows from the proof of Theorem 2.8 that they are the same, so it must be a solution, that is, an ow-solution as well as an ew-solution. Specifically, it can be expressed as

$$
x(t)= \begin{cases}x_{1}(t), & t \in[2 n-2,2 n-1) \forall n \in Z, \\ x_{2}(t), & t \in[2 n-1,2 n) \forall n \in Z,\end{cases}
$$

where

$$
\begin{array}{r}
x_{1}(t)=(-1)^{n} \frac{2}{3 \pi^{2}}(2 t-4 n+3)-\frac{2}{\pi^{2}} \cos \frac{\pi}{2} t+\frac{2}{\pi^{2}} \sin \frac{\pi}{2} t, \quad t \in[2 n-2,2 n-1) \forall n \in Z, \\
x_{2}(t)=(-1)^{n} \frac{4}{3 \pi^{2}}+(-1)^{n+1} \frac{2}{3 \pi^{2}}\left((t-2 n)^{2}+(t-2 n+1)^{2}\right)-\frac{2}{\pi^{2}} \cos \frac{\pi}{2} t+\frac{2}{\pi^{2}} \sin \frac{\pi}{2} t, \\
t \in[2 n-1,2 n) \forall n \in Z .
\end{array}
$$
$n \in z$.

It is easy to check that $x(t)$ is an almost-periodic solution of (1.2) such that $x(n)=x_{n}$

(ii) We show that $\left\{x_{n}\right\} \in F_{1}$ (resp., $F_{2}$ ) is not unique solution of (2.5) (resp., (2.6)). Let

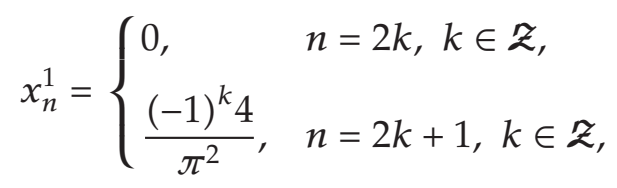

$$
\begin{aligned}
& \widehat{x}_{n}^{1}= \begin{cases}0, & n=2 k, k \in \mathcal{Z}, \\
\frac{(-1)^{k-1} 2}{\pi^{2}}, & n=2 k+1, k \in \mathfrak{Z} .\end{cases}
\end{aligned}
$$

Obviously, $\left\{x_{n}^{1}\right\} \in F_{1}$ is another solution of (2.5). 
Let $\left\{y_{n}^{1}\right\}=\{0\}$ and

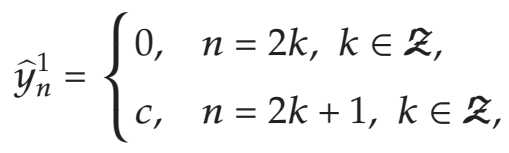

where $c$ is an arbitrary constant, then it is clear that $\left\{y_{n}^{1}\right\} \in F_{2}$ is another solution of (2.6).

The following example shows that ow-solutions and ew-solutions of (1.2) are not equivalent.

Example 3.2. As [6], let $f(t)=(-1)^{n}(t-2 n+1), t \in[2 n-2,2 n)$, for all $n \in \mathcal{Z}$, then $f(t) \in \mathcal{A} P(R)$, setting $g(t)=f(t) / 2$, then $g(t) \in \mathcal{A} D(R)$ and $f(t)=g(t)-g(t-2)$, for all $t \in R$, that is, the assumption $(\mathrm{H})$ holds. By simple calculation, we can obtain $f_{2 n}^{(1)}=f_{2 n}^{(2)}=(-1)^{n} 1 / 3, f_{2 n-1}^{(1)}=$ $(-1)^{n} 1 / 6, f_{2 n-1}^{(2)}=(-1)^{n+1} 1 / 6, g_{2 n}^{(1)}=g_{2 n}^{(2)}=(-1)^{n} 1 / 6, g_{2 n-1}^{(1)}=(-1)^{n} 1 / 12, g_{2 n-1}^{(2)}=(-1)^{n+1} 1 / 12$, $g_{2 n}=h_{2 n} / 2=(-1)^{n} 1 / 3, h_{2 n-1}=g_{2 n-1}=0$.

(i) We construct the almost-periodic ow-solution of (1.2) as the proof of Theorem 2.6. Let

$$
\begin{aligned}
& x_{n}= \begin{cases}0, & n=2 k, k \in z, \\
\frac{(-1)^{k} 1}{3}, & n=2 k+1, k \in z,\end{cases} \\
& \widehat{x}_{n}= \begin{cases}0, & n=2 k, k \in Z, \\
\frac{(-1)^{k+1} 1}{6}, & n=2 k+1, k \in Z,\end{cases}
\end{aligned}
$$

then $\left\{x_{n}\right\} \in F_{1}$ is the solution of (2.5). Calculate $\widehat{\omega}_{2 n}, \omega_{2 n}$ as the formulas mentioned above, we obtain $\omega_{2 n}=(-1)^{n} 1 / 3, \widehat{\omega}_{2 n}=(-1)^{n-1} 1 / 6$. Obviously, $\left\{\omega_{2 n}\right\},\left\{\widehat{\omega}_{2 n}\right\} \in \operatorname{ADS}(R)$.

Define the ow-solution $x(t)$ as

$$
x(t)= \begin{cases}x_{1}(t), & t \in[2 n, 2 n+1) \forall n \in Z, \\ x_{2}(t), & t \in[2 n-1,2 n) \forall n \in Z,\end{cases}
$$

where

$$
\begin{aligned}
& x_{1}(t)=(-1)^{n} \frac{1}{6}+(-1)^{n} \frac{1}{12}(2 t-4 n-1)\left(2-(t-2 n)^{2}+(t-2 n)\right), \quad t \in[2 n, 2 n+1), \\
& x_{2}(t)=(-1)^{n} \frac{7}{12}(t-2 n)+(-1)^{n} \frac{1}{4}(t-2 n)^{2}, \quad t \in[2 n-1,2 n) .
\end{aligned}
$$

It is easy to check that $x(t)$ is an almost-periodic ow-solution of (1.2). Since $\left\{x_{2 n}\right\}$ is not solution of (2.17), it follows from Theorem 2.10 that $x(t)$ is not solution of (1.2) and consequently, $x(t)$ is not an ew-solution of (1.2).

(ii) Similarly to (i), by Theorem 2.7 , we construct the almost-periodic ew-solution of (1.2).

Let $\left\{y_{n}\right\}=\{0\}$ and

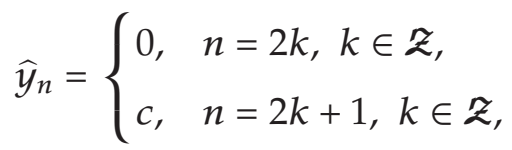


where $c$ is an arbitrary constant, then $\left\{y_{n}\right\} \in F_{2}$ is the solution of (2.6). Calculate $\widehat{\omega}_{2 n+1}, \omega_{2 n+1}$ as the formulas mentioned above, we obtain $\omega_{2 n-1}=(-1)^{n+1} 1 / 6, \widehat{\omega}_{2 n+1}=(-1)^{n+1} 1 / 12$. Obviously, $\left\{\omega_{2 n-1}\right\},\left\{\widehat{\omega}_{2 n-1}\right\} \in \mathcal{A} D \mathcal{S}(R)$.

Define the ew-solution $y(t)$ as

$$
y(t)= \begin{cases}y_{1}(t), & t \in[2 n-2,2 n-1) \forall n \in Z, \\ y_{2}(t), & t \in[2 n-1,2 n) \forall n \in Z,\end{cases}
$$

where

$$
\begin{aligned}
& y_{1}(t)=(-1)^{n} \frac{1}{12}(2 t-4 n+3)\left((t-2 n)^{2}+3(t-2 n)+2\right), \quad t \in[2 n-2,2 n-1), \\
& y_{2}(t)=(-1)^{n} \frac{1}{4}\left((t-2 n)^{2}+t-2 n\right), \quad t \in[2 n-1,2 n) .
\end{aligned}
$$

It is easy to verify that $y(t)$ is an almost-periodic ew-solution of (1.2). Since $\left\{y_{2 n}\right\}$ is not solution of (2.17), it follows from Theorem 2.10 that $y(t)$ is not solution of (1.2) and consequently, $y(t)$ is not an ow-solution of (1.2).

\section{Acknowledgment}

The research is supported by the NSF of China no. 10671047.

\section{References}

[1] K. L. Cooke and J. Wiener, "Retarded differential equations with piecewise constant delays," Journal of Mathematical Analysis and Applications, vol. 99, no. 1, pp. 265-297, 1984.

[2] S. M. Shah and J. Wiener, "Advanced differential equations with piecewise constant argument deviations," International Journal of Mathematics and Mathematical Sciences, vol. 6, no. 4, pp. 671-703, 1983.

[3] G. Seifert, "Second-order neutral delay-differential equations with piecewise constant time dependence," Journal of Mathematical Analysis and Applications, vol. 281, no. 1, pp. 1-9, 2003.

[4] D. X. Piao, "Almost periodic solutions of neutral differential difference equations with piecewise constant arguments," Acta Mathematica Sinica, vol. 18, no. 2, pp. 263-276, 2002.

[5] H.-X. Li, "Almost periodic solutions of second-order neutral delay-differential equations with piecewise constant arguments," Journal of Mathematical Analysis and Applications, vol. 298, no. 2, pp. 693-709, 2004.

[6] H.-X. Li, "Almost periodic weak solutions of neutral delay-differential equations with piecewise constant argument," Nonlinear Analysis: Theory, Methods \& Applications, vol. 64, no. 3, pp. 530-545, 2006.

[7] E. A. Dads and L. Lhachimi, "New approach for the existence of pseudo almost periodic solutions for some second order differential equation with piecewise constant argument," Nonlinear Analysis: Theory, Methods \& Applications, vol. 64, no. 6, pp. 1307-1324, 2006.

[8] R. Yuan, "Pseudo-almost periodic solutions of second-order neutral delay differential equations with piecewise constant argument," Nonlinear Analysis: Theory, Methods \& Applications, vol. 41, no. 7-8, pp. 871-890, 2000.

[9] R. Yuan, "Existence of almost periodic solutions of second order neutral delay differential equations with piecewise constant argument," Science in China Series A, vol. 41, no. 3, pp. 232-241, 1998.

[10] Z. Li and M. He, "The existence of almost periodic solutions of second order neutral differential equations with piecewise constant argument," Northeastern Mathematical Journal, vol. 15, no. 3, pp. 369-378, 1999.

[11] C. Zhang, Almost Periodic Type Functions and Ergodicity, Science Press/Kluwer Academic Publishers, Beijing, China, 2003. 